\title{
Significant physical improvement of spinal cord injured patients after neural stem cell transplantation
}

\begin{abstract}
In this case report, we provided four patient cases and their significant physical improvement after human fetal neural stem cell (NSC) transplantation. The spinal cords of the patients were severely injured during accidents, and after traditional treatment in hospitals, they physically improved very little and slowly. After 8 to 14 times of NSC transplantation, they improved significantly. These data demonstrated that human fetal NSC therapy is both efficient and safe.
\end{abstract}

Keywords: spinal cord injury, human fetal neural stem cells, stem cell therapy, physical improvements
Volume 2 Issue I - 2017

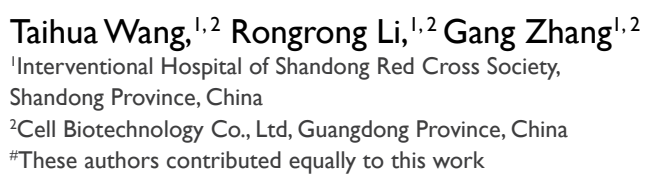

Correspondence: Taihua Wang, Interventional Hospital of Shandong Red Cross Society, Jinan, Shandong Province, China, 250000,Email drwangtaihua@hotmail.com

Gang Zhang, Interventional Hospital of Shandong Red Cross Society, Jinan, Shandong Province, China, 250000,

Email sdzbzhanggang@I63.com,gang_zhang@stemceltech.com

\section{Introduction}

Stem cell transplantation therapy has been controversial during the past decades due to concerns about its safety and efficacy. ${ }^{1-3}$ The main concerns focused on the tumor formation and immune rejection following the use of allogeneic stem cells. Here, we reported four patient cases whose spinal cords were severely injured by car accidents or other accidents. We isolated human fetal neural stem cells (NSCs) from aborted fetuses and cultured them in DMEM/F12+B27 $(1 \mathrm{X})+\mathrm{bFGF}(20 \mathrm{ng} / \mathrm{ml})+\mathrm{EGF}(20 \mathrm{ng} / \mathrm{ml})+\mathrm{N} 2(1 \mathrm{X})(\mathrm{Gibco}) .{ }^{4}$ All the treatments were performed according to the guidelines established in the Interventional Hospital of Shandong Red Cross Society for patients and the human fetal NSCs were isolated with the consent of their parents. We proliferated the NSCs and transplanted them into the patients via lumbar puncture, each time, the transplanted NSCs were about $4 \times 10^{7}$ cells. After 8 to 14 times of transplantation therapy, all the four patients were physically improved significantly.

\section{Patient cases}

\section{Patient case I}

This patient was severely injured on July 14, 2009 in a car accident, and the lower part of his body was paralyzed from then on. His 12th thoracic vertebrate was comminuted bursting fracture, and his body under the 12 th thoracic vertebrae was in imperceptions. Gradually yet slowly, after about 3 months, he recovered the consciousness of the lower part of his body. At that time, his spinal nerves were injured very severely. After rehabilitation training, he could walk very slowly with the help of two crutches. At that time, he was at a rehabilitation center in Henan Province, China. His doctors were very surprised that he could stand up, and believed that he already recovered to his best state, and did not need any further therapy. But he had less sense of the lower part of his body, and the muscular tension was very high, and the muscles of the lower part of his body were numb. In addition, he had urinary and fecal incontinence.
Generally, he would have bowel movement once every three or four days, and did not have urinary control. After rehabilitation training, he could walk very slowly with the help of two crutches, but with very severely quivering hands and feet. When he wanted to stand up, he needed to use his two hands to support his body with lots of strength, and his both legs would quiver 2 to 3 minutes until he could control his body. Furthermore, when he was surprised, his legs would quiver for a long time and he could not walk before he recovered.

After a total of 10 times transplantations of human fetal NSCs in our hospital, he could walk slowly without the help of the two crutches. He regained his regular bowel movements and urinary control. In addition, the muscular tension of the lower part of his body decreased greatly. Basically, he could live his everyday life without the crutches, and also, he could take the train from Henan province to Shandong province to and fro (about 600 kilometers one way) by himself and did not need his family members to accompany him.

During the recent several years, his recovery was very well, and his physical state improved greatly. These improvements included the atrophy of his leg muscles, urinary and bowel movement control, and he could stand up without external help, and walk freely. He did not quiver and his muscular tension was not high, and so on. Moreover, before he accepted the NSC therapy, every night he turned around his body frequently and could not sleep well. Yet after the therapy, he slept very well. At present, he even could ride bicycle and motorcycle. As he said, "if some people did not know I had the car accident before, they could not see that I had been injured severely".

\section{Patient case 2}

The second patient was aged 32 , at about $5 \mathrm{pm}$ of October $23 \mathrm{rd}$, 2009, he accidentally fell down from the roof of a Chinese house, which is about three meters high, and his back hit the ground and he was severely injured. He lost his consciousness for about 3 minutes. After the accident, there were many skin abrasions on his whole body, 
and he felt that his back waist was very pain. In addition, the lower part of his body was numb and could not be moved. He called the emergency phone, and was sent to the local hospital.

When he was at the hospital, he got his consciousness back, but his both legs still could not move. The MRI examination of his chest showed that his thoracic vertebrae T11 had a compression fracture and was dislocated. After emergency treatment at the local hospital, he was transferred to a better-conditioned hospital. After complete examination at the hospital, he was treated with "decompression bone grafting and internal fixation" surgery, and the whole procedure was well done. After he left the hospital, he sequentially got several different therapies, including rehabilitation at different hospitals and acupuncture, and so on, but their effects were not very well.

In February of 2013, he was treated at our hospital with NSC therapy, and after a total of 12 times NSC transplantation treatments, his physical condition was improved significantly and he could feel his waist was strengthened. At present, he could sit up freely, but still could not stand up. He also could feel his urine and feces, but still could not control them. The muscular tension of his both legs was improved obviously. And he could crawl, and walk with the help of walking aides. In addition, his double arms could move freely.

\section{Patient case 3}

The third patient was 24 years old when the accident took place. His head, face and the back of his chest were injured in a car accident at April 1st of 2012. Following the accident, he was in a coma for about 30 minutes. After he recovered from the coma, he felt his chest was painful and stuffy. In addition, his both legs had limited movement, and his sensation was lost under the xiphoid. He was unconscious, could not control his urine and bowel movements, without hemoptysis, but with abdominal pain. He was urgently sent to the local hospital, and after CT examination, it was found that several of his ribs were fractured, and his breastbone was broken. He also had double side hydro-pneumothorax, traumatic wet lung, thoracic fractures and subdural hematoma.

At the hospital, after all the necessary examinations, he was treated with anti-inflammatories, hemostatics, analgesics and fluid infusion. On April 6th, his chest was examined with CT, and found there was lots of fluid accumulated in his thoracic cavity. On April 6th and 7th, 2012, he was treated with bilateral pleural closed drainage surgery, and after the treatment, his chest symptoms were improved. And at April 17th, 2012, he was treated for 5th thoracic vertebrae fracture dislocation, with posterior open reduction and pedicle screw fixation under general anesthesia, and treated accordingly after the surgeries. At that time, his both legs could move, at his right side, his senses decreased under thoracic vertebrae 4th, and at his left side, his senses under the 5 th thoracic vertebrae degenerated. In some sense, he had some urine and bowel control although the control was very weak.

From November of 2013 on, after a total of 8 times NSC treatments delivered in our hospital, he began to feel his waist was strengthened. Now, he could sit up freely, but could not stand up. He could feel his urine and feces, but could not control them. The muscular tension of his both legs was lower than before, but his legs were still sweating a lot. In addition, his sensory level moved downward slightly up to now.

\section{Patient case 4}

The fourth patient was 40 years old when he was injured during a car accident on August 2nd of 2007. He was immediately sent to the Hospital of Jimo City. The CT examination of his head showed that his right temporal lobe brain was with contusion, and subarachnoid hemorrhage. The CT of his chest showed that his 4-6 thoracic vertebrate were fractured. In addition, the X-ray examination of his chest showed that some of his left ribs were broken, and with hydropneumothorax. On his right side, there was a little pleural effusion. The diagnosis was, "cerebral contusion and laceration, scalp avulsion, thoracic fractures of T4-T6 and spinal cord injury, and bilateral hemopneumothorax".

He was treated according to his symptoms, including suturing the head injury, indwelling urinary catheter, the closed drainage of the right side chest, transfusion of $1000 \mathrm{ml}$ whole blood during the surgery, and so on. He regained his consciousness after 5 hours of coma, but his both legs could not move, and he could not feel his urine and feces as well. On Aug 24 of the same year, under the general anesthesia condition, he was treated with vertebrae resection of his thoracic vertebrae T5 and T6, plus bone graft fusion and screw rod internal fixation. After the surgery, he was also treated with anti-inflammation, dehydration, hyperbaric oxygen and functional rehabilitation, etc.

In September of the same year, his right foot toes could move slightly, and his right leg began to have the sense of touch. After 6months of hospitalization, his indwelling urinary catheter was removed, and he also had better urine and bowel control, but still not sensitive. In addition, his right leg could bend and stretch slightly, but he still could not sit up. And then, he went back to his home and continued his rehabilitation. Until February of 2008, he could feel his urine and feces sensitively, but he could not hold back his urine. At the end of 2008, he felt his waist was strengthened, and he could sit up by himself strenuously.

From the January of 2010 on, he was treated with a total of 14 times NSC transplantations in our hospital. Afterward, he began to feel his waist was strengthened significantly. Now, he could sit up freely, but still could not stand up. He could feel his urine and feces, but still could not control them. His left leg could move levelly, but could not lift up. His right leg could lift up slightly, but could not overcome the resistance. The muscular tension of his both legs was relatively high, and he had sensory disturbance under his xiphoid level. In addition, his both arms could move freely.

\section{Conclusion}

As for the safety of stem cell transplantation, during the past 12 years, we have treated about 10 thousand patients with stem cell transplantation, and so far, no tumor formation has been observed in any patients (unpublished data). These data vividly demonstrate that stem cell transplantation is safe for curing human diseases. In most of the patient cases, we used human fetal NSCs to treat the patients with spinal injuries, Parkinson disease, and so on. In our another report, we used human neural stem cells to treat 17 Parkinson disease patients, after three months following-up visit, we found that the effective rates of stem cell transplantation were $82.4 \%$ in the contralateral sites and $64.7 \%$ in the ipsilateral sites, respectively. ${ }^{5}$ These clinical investigations proved that the transplanted NSCs could regenerate in the patients manifested by the physical improvements of the treated patients. This is a great discovery clinically, and overturns the hypothesis that neural cells cannot regenerate in vivo, and lays important foundations for neural regenerative medicine. Furthermore, stem cell transplantation plays significant roles in sub-health patients 
and human preventive medicine. Through regulating immune system and other unknown reasons, the transplanted stem cells can regulate the function of human immune system, and the effectiveness is above 95\% (unpublished data).

\section{Acknowledgements}

None.

\section{Conflict of interest}

The author declares no conflict of interest.

\section{References}

1. Freed CR, Greene PE, Breeze RE, et al. Transplantation of embryonic dopamine neurons for severe Parkinson's disease. $N$ Engl $J$ Med. 2001;344(10):710-719.
2. Hagell P, Piccini P, Björklund A, et al. Dyskinesias following neural transplantation in Parkinson's disease. Nat Neurosci. 2002;5(7):627628.

3. Olanow CW, Goetz CG, Kordower JH, et al. A double-blind controlled trial of bilateral fetal nigral transplantation in Parkinson's disease. Ann Neurol. 2003;54(3):403-414.

4. Conti L, Pollard SM, Gorba T, et al. Niche-independent symmetrical self-renewal of a mammalian tissue stem cell. PLoS Biol. 2005;3(9):e283.

5. Taihua Wang, Yanzhong Xue, Xiuxiu Li, et al. Computer assisted stereotactic transplantation of human neural stem cells in Parkinson's disease patients; 2017. 